\title{
A finite element model for thermomechanical analysis in casting processes
}

\author{
D. CELENTANO, S. OLLER and E. OÑATE
}

International Center for Numerical Methods in Engineering, E.T.S. d'Enginyers de Camins, Canals i Ports, Universitat Politècnica de Catalunya, Gran Capità s/n, Mòdul C1, 08034 Barcelona, Spain

\begin{abstract}
This paper summarizes the recent work of the authors in the numerical simulation of casting processes. In particular, a coupled thermomecharical model to simulate the solidification problem in casting has been developed $[7,8,9]$. The model, based on a general isotropic thermoclasto-plasticity theory and formulated in a macroscopical point of view, includes generalized phase-change effects and considers the different thermomechanical behaviour of the solidifying material during its evolution from liquid to solid. For this purpose, a phase-change variable, plastic evolution equations and a temperature-dependent material constitutive law have been defined. Some relevant aspects of this model are presented here.

Full thermomechanical coupling terms have been considered as well as variable thermal and mechanical boundary conditions: the first are due to air gap formation, while the second involve a contact formulation.

Particular details concerning the numerical implementation of this model are also mentioned. An enhanced staggered scheme, used to solve the highly non-linear fully coupled finite element equations, is proposed. Furthermore, a proper convergence criterion to stop the iteration process is adopted and, although the quadratic convergence of Newton-Rapshon's method is not achieved, several numerical experiments demonstrate reasonable convergence rates [?].
\end{abstract}

Finally, an experimental cylindrical casting test problem, including phase-change phenomena, temperaturedependent constitutive properties and contact effects, is analyzed. Numerical sesults are compared with some laboratory measurements.

\section{INTRODUCTION.}

The full thermomechanical behaviour of bodies with thermal and mechanical temperaturedependent material properties that change their temperature during a certain process is of great practical importance in many engineering situations. In particular, a major consideration is the formation of cracks due to induced thermal stress field in casting processes. Although an analytical investigation into thermal stress development has been attempted by many researchers ${ }^{[3-6]}$, it has been long recognized that the use of numerical methods of solution are necessary to consider realistic complex problems.

For this purpose, thermomechanical formulations with internal variables governed by rate equations (that can nowadays be considered well established ${ }^{[1,2]}$ ), theoretical extensions, implementations into computer programs and numerical analyses have been also carried out by many researchers (i.e. see $[3-6]$ ). Some of these models have been used to simulate solidification processes (see $[4-6]$ and references therein). Nevertheless, the problem has many complex aspects that are usually difficult to deal with:

- the equilibrium and energy equations are coupled. Consequently, a robust and efficient numerical strategy is crucial for solving the highly non-linear finite element equations,

- a constitutive model which can represent the liquid, mushy and solid phases is necessary for the casting,

- different kind of materials are usually involved in solidification processes, 
- thermal and mechanical variable boundary conditions must be taken into account. This fact implies the consideration of a pressure/gap-dependent convection-radiation model and a contact-friction formulation, respectively,

- latent heat effects introduces oscillations in the algorithms,

- an accurate residual stress evaluation has to be performed,

- microscopical effects may be considered.

A general thermoelasto-plastic model taking into account many of previous aspects has been presented in References [7,8]. In this paper, important details of this model and some hypothesis assumed in its formulation are discussed in Section 2. Furthermore, a thermomechanical contact model (that includes a gap-dependent convection-radiation model and a mechanical contact formulation), is also considered. A complete description of this last model can be found in Reference [9]. As a first approach to the problem, friction effects will be neglected in the present work.

Although the weak form and the finite element formulation of this model is derived in Reference [9] (where special attention is devoted to "non-classical" terms containing phase-change, thermomechanical coupling and contact effects), the numerical strategy used to solve the non-linear system of equations is presented in Section 3 .

Finally, in Section 4, an experimental cylindrical casting test problem is analyzed and numerical results are contrasted with some laboratory measurements.

\section{THERMOELASTO-PLASTIC MODEL.}

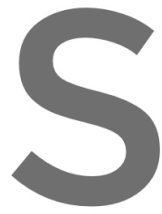

a) Basic definitions.
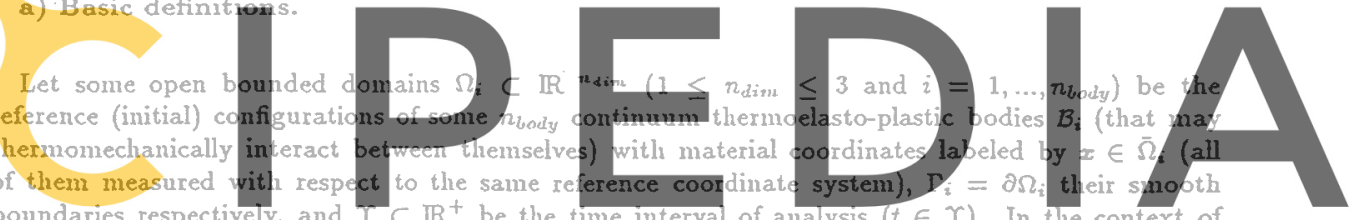

boundaries respectively, and $I \subset \mathbb{R}^{+}$be the time interval of analysis $(t \in \Upsilon)$. In the context of

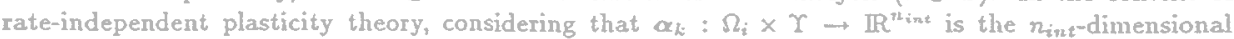

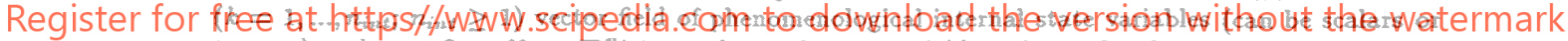
tensors) and $q_{k}: \Omega_{i} \times \Upsilon \rightarrow \mathbb{R}^{n_{i n t}}$ are the corjugate variables of $\alpha_{k}$, the thermoplastic behaviour of the solid is governed by a state function $F\left(q_{k}\right): \mathbb{R}{ }^{\text {thint }} \rightarrow \mathbb{R}$, called the yield function (assumed strictly convex and, for simplicity, defined in terms of a unique smooth function) such that no plastic evolutions occurs when $F<0$ [1]. Therefore, the thermoelastic admissible domain (also assumed convex) $\mathbb{I E}^{\text {te }}$ is defined as $[1]$ :

$$
\mathbb{I E}^{t e}=\left\{q_{i} \in \mathbb{R}^{n_{i n t}} \mid F\left(q_{k}\right)<0\right\},
$$

and the thermoplastic one is:

$$
\operatorname{IE}^{t p}=\left\{q_{k} \in \mathbb{R}^{n_{i n t}} \mid F\left(q_{k}\right)=0\right\}=\partial \mathrm{IE}^{t c} .
$$

Further, the assumption of the principle of naximum plastic dissipation leads to an associate constitutive model characterized by the following plastic evolution equations [1]:

$$
\dot{\boldsymbol{\alpha}}_{k}=\frac{\partial F}{\partial \boldsymbol{q}_{k}} \dot{\lambda} \quad \text { in } \Omega_{i} \times \Upsilon
$$

together with the load-unload Kuln-Tucker conditions and the Prager's consistency condition [1]. In equation $(2.2), \dot{\lambda}$ is the plastic consistency paraneter [1]. 
In particular, one possible option for equation (2.2) is to choose $\left(\alpha_{1}=\epsilon^{p}, \alpha_{2}=C^{p}, \alpha_{3}=\eta^{p}\right)$, where $\epsilon^{p}: \Omega_{i} \times \Upsilon \rightarrow \mathbb{R}^{n_{\text {dim }}} \times \mathbb{R}^{n_{\text {dim }}}$ is the plastic strain second-rank tensor, $C^{p}: \Omega_{i} \times \Upsilon \rightarrow \mathbb{R}$ is the plastic hardening function and $\eta^{p}: \Omega_{i} \times \Upsilon \rightarrow \mathbb{R}$ is the plastic entropy, with evolution equations [3]:

$$
\begin{aligned}
& \dot{\boldsymbol{\epsilon}}^{\prime}=\frac{\partial F}{\partial \sigma} \dot{\lambda} \quad \text { in } \Omega_{i} \times \Upsilon, \\
& \dot{\mathcal{C}}^{p}=\frac{\partial F}{\partial q_{C r}} \dot{\lambda} \quad \text { in } \Omega_{i} \times \Upsilon, \\
& \dot{\eta}^{p}=\frac{\partial F}{\partial T} \dot{\lambda} \quad \text { in } \Omega_{i} \times \Upsilon,
\end{aligned}
$$

where $\sigma$ (Cauchy stress tensor), $q C$ r and $T$ (temperature) are the conjugate variables of $\epsilon^{p}, C^{p}$ and $\eta^{p}$, respectively. The yield function is written in this case as $F\left(\sigma, q_{\mathcal{C} p}, T\right):\left(\mathbb{R}^{n_{\text {sim }}} \times \mathbb{R}^{n_{\text {sim }}}\right) \times \mathbb{R} \times \mathbb{R}^{+} \rightarrow$ $\mathbb{R}$, with $\mathbb{E}^{t_{e}}$ and $\mathbb{E E}^{t_{p}}$ defined as in equations (2.1).

Nevertheless, a more simpler model takes place if the evolution equation for $\mathcal{C}^{p}$ is assumed to be defined in terms of $\dot{\epsilon}^{p}$ as $[7,8\}$ :

$$
\dot{C}^{p}=H_{C P} \dot{\lambda} \text { in } \Omega_{i} \times \Upsilon \text {, }
$$

where $H_{C r}(T)=h_{c r}(T) \sigma: R$ is the plastic hardening coefficient, $h_{\mathcal{C}}(T)$ is the plastic hardening modulus and $R=\frac{\partial F}{\partial \sigma}$ is the flow potential. Consequently, $F$ is redefined once more as $F\left(\sigma, C^{y}, T\right)$ : $\left(\mathbb{R}^{n_{\text {dim }}} \times \mathbb{R}^{n_{\text {dim }}}\right) \times \mathbb{R} \times \mathbb{R}^{+} \rightarrow \mathbb{R}$, with:

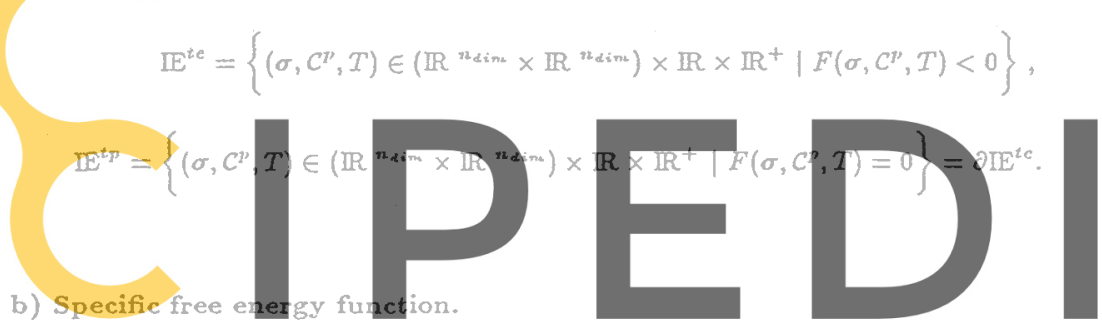

b) Specific free energy function.

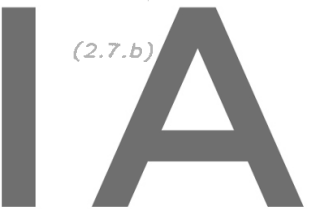

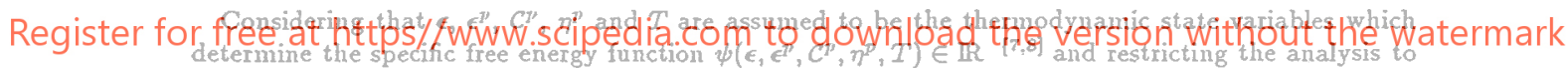
the case of thermoelasto-plastic isotropic response, the function $\psi$ is formulated as:

$$
\psi=\hat{\psi}_{t e}\left(\epsilon-\epsilon^{p}, T\right)+\hat{\psi}_{p}\left(C^{p}, \eta^{p}, T\right)+\hat{\psi}_{p c}(T) \text { in } \Omega_{i} \times \Upsilon,
$$

where $\psi_{t e}, \psi_{t p}$ and $\psi_{p c}$ are the thermoelastic, thermoplastic and phase-change parts of $\psi$, respectively. It should be noted that equation (2.8) is a decoupled form of defining the specific free energy $\psi$. The thermoelastic part $\psi_{t e}$ is written as:

$$
\begin{aligned}
& \psi_{t e}=\hat{\psi}_{t e}\left(\epsilon-\epsilon^{p}, T\right)=\frac{1}{2 \rho_{o}}\left(\epsilon-\epsilon^{p}\right): \Phi^{s}:\left(\epsilon-\epsilon^{p}\right)-\frac{1}{\rho_{o}} \beta^{*}:\left(\epsilon-\epsilon^{p}\right)\left(T-T_{o}\right)+ \\
& +\psi_{o}-\eta_{o}\left(T-T_{o}\right)+\frac{1}{\rho_{o}} \sigma_{o}:\left(\epsilon-\epsilon^{p}\right)+c^{*}\left(T-T_{o}\right)-c^{*} T \ln \frac{T}{T_{o}} \text { in } \Omega_{i} \times \Upsilon,
\end{aligned}
$$

the thermoplastic part is:

$$
\psi_{t p}=\hat{\psi}_{t, p}\left(\mathcal{C}^{p}, \eta^{p}, T\right)=\hat{\psi}_{p}\left(\mathcal{C}^{p}\right)-T \eta^{y} \quad \text { in } \Omega_{i} \times \Upsilon
$$

and the phase-change part is:

$$
\psi_{p e c}=\hat{\psi}_{p c c}(T)=-\int_{T_{o}}^{T} A_{y c}(\theta) d \theta \text { in } \Omega_{i} \times \Upsilon .
$$


The subscript o denotes the initial state of the different variables and the superscript $s$ indicates secant thermomechanical properties. $\rho$ is the density, $\eta$ is the entropy, $c$ is the specific heat capacity, $\mathbb{C}$ is the elastic tangent constitutive fourth-rank (isothermal) tensor and $\beta$ is the tangent conjugate of thermal dilatation second-rank tensor. Further, $\psi_{p}$, is the purely plastic part of $\psi$ (assumed known) and $A_{p c}(T)=\int_{T_{0}}^{T} \frac{L}{\theta} \frac{\partial f_{T_{c}}(\theta)}{\partial \theta} d \theta$ ( $\theta$ is a dummy variable).

With the present definition of $\psi$ the secant and tangent constitutive laws, the entropy function, the specific heat capacity and the thermoplastic coupling term can be derived. Details of such derivations can be found in Reference [9].

\section{c) Constitutive tensor.}

During solidification, the material in liquid state becomes solid, which means that a qualitative change in its thermomechanical properties is produced. Therefore, it is necessary to take this fact into account in the constitutive tensor written as,

$$
\mathbb{C}=\mathbb{C}_{\text {mol }}+\rho_{T}(T) \mathbb{C}_{d e t}
$$

where $\mathbb{C}_{* \infty}$ and $\mathbb{C}_{\text {tev }}$ are the volumetric and deviatoric parts of $\mathbb{C}$ respectively, and $\rho_{T} \in[0,1]$ is the "phase-change" variable.

With this definition of $\mathbb{C}$, both the classic constitutive law of a liquid in rest $\left(\sigma^{\prime}=0\right.$, i.e. $\sigma$ results the hydrostatic stress tensor), and that corresponding to a solid can be represented.
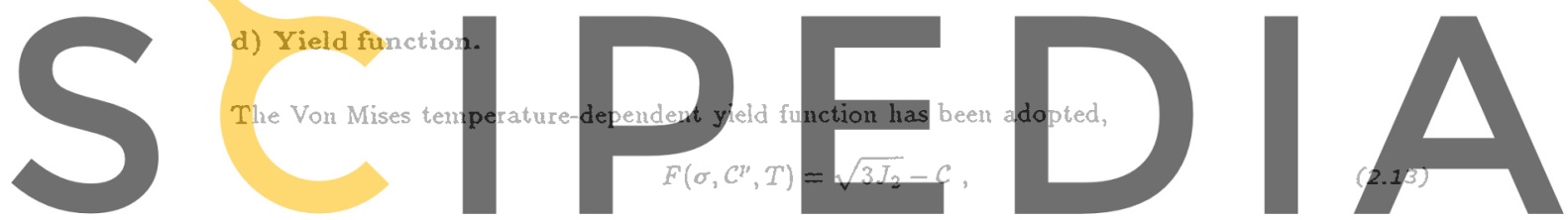

where $J_{2}=\frac{1}{2} \sigma^{\prime}: \sigma^{\prime}$ is the second invariant of the deviatoric stress tensor $\sigma^{\prime}$ and $C: \Omega_{i} \times \Upsilon \rightarrow \mathbb{R}$ is Register for free at hiptening function definediby: $c\left(C^{1}, T\right)=c^{t h}(T)+c^{1 x}$

where $\mathcal{C}^{\text {th }}(T): \Omega_{i} \times \Upsilon \rightarrow \mathbb{R}^{+}$is the thermal hardening function (also assumed to be smooth function of $T$ ) related to the cohesion of the material (even in the liquid plase).

It should be noted that $R=\frac{\sqrt{3}}{2 \sqrt{J_{2}}} \sigma^{\prime}$ is indeterminate when $\sigma^{\prime}=0$. However, as $C^{t h}>0$, it can be observed that $F<0$ for this particular situation. Therefore, a purely elastic belaviour of the material $(\dot{\lambda}=0)$ is considered for this case.

h) Specific heat capacity.

In phase-change problems, the following general definition is adopted for $c$ [9]:

$$
c=-T \frac{\partial^{2} \psi}{\partial T^{2}}-L \frac{\partial f_{\mu c}(T)}{\partial T} \quad \text { in } \Omega_{i} \times \Upsilon,
$$

where $\omega: \Omega_{i} \times \Upsilon \rightarrow \mathbb{R}$ is the specific internal energy, $L$ is the latent heat released in a freezing problem (or absorbed in a melting one) and $f_{p c}$ is the "phase-change" function. In an isothermal plase-change problem $f_{p c}=H\left(T-T_{m}\right)$, with $T_{m}$ being the melting temperature and $H$ the Heaviside function. When the phase-change occurs in a range of temperatures $\left(T_{l}-T_{*}\right)$, where $T_{*}$ and $T_{l}$ are the solidus and liquidus temperature respectively, $f_{p c}=f$ such that, 


$$
f(T)= \begin{cases}0 & ; \forall T<T . \\ 0 \leq g(T) \leq 1 & ; T_{s} \leq \forall T \leq T_{l} \\ 1 & ; \forall T>T_{i} .\end{cases}
$$

The function $g(T)$ may be obtained using a microstructure model. However, from a macroscopical point of view assumed in this paper, the simplest choice for $g(T)$ is the linear one [9].

It should be noted that the classical definition of $c{ }^{[1]}$ is recovered in those regions where the temperature derivative of $f_{p c}$ is zero. Equation (2.15) describes the generalized phase-change problem. A further generalization takes place when two or more phase-changes $\left(n_{p c} \geq 2\right)$ can occur. For this case, the term $L \frac{\partial f_{r c}}{\partial T}$ must be replaced by $\sum_{j=1}^{n} L_{j} \frac{\partial f_{p c_{j}}}{\partial T}\left(L_{j}\right.$ and $f_{p c_{j}}$ are the latent heat and phase-change function associated with the $\mathrm{j}$-th phase-change, respectively) in equation (2.15).

As mentioned above, considering the present definition of $\psi$ given in Section $2 . b$, the expression of $c$ can be derived [?].

\section{SOLUTION STRATEGY: STAGGERED SCHEME.}

Details of the weak form and finite element formulation of this model can be found in Reference [9]. The numerical solution at time $t+\Delta t$ of the resulting finite element coupled thermo-mechanical equations (the solution is assumed known at time $t$ ) lhas been attempted via a staggered scheme of type:
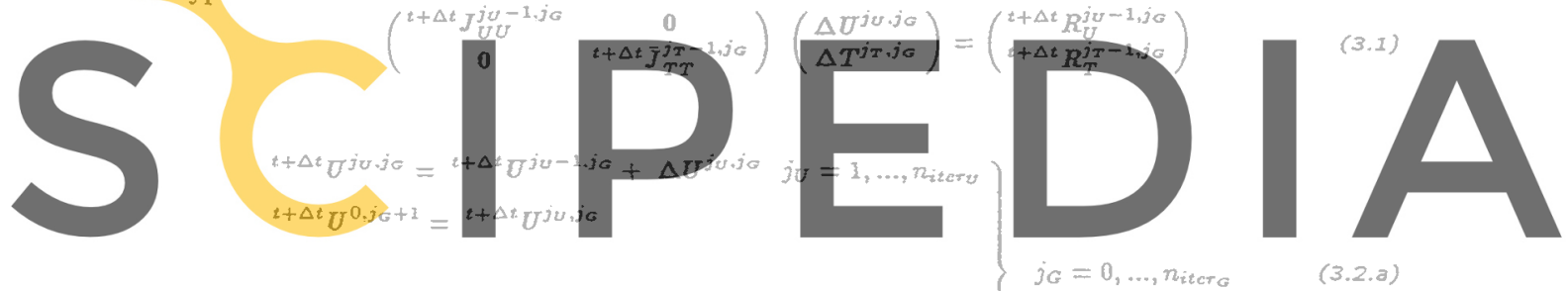

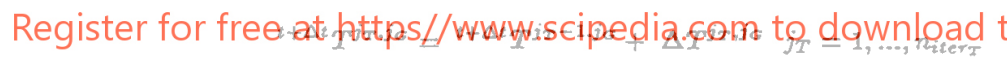

(3.2.a)

$$
\begin{aligned}
& t+\Delta t T^{0, j G+1}=t+\Delta t T^{j T \cdot j G} \\
& \left.\begin{array}{l}
{ }^{t+\Delta t} U^{0.0}={ }^{t} U \\
{ }^{t+\Delta t} T^{0.0}={ }^{t} T
\end{array}\right\}
\end{aligned}
$$

where $j_{v}, j_{T}$ and $j_{G}$ are the iteration indexes of the mechanical, thermal and thermomechanical problems, respectively. Further, the matrix notation is the following: $J_{U U}$ : mechanical jacobian matrix, $J_{T T}$ : thermal jacobian matrix, $U$ : nodal displacement vector, $T$ : nodal temperature vector, $\boldsymbol{R}_{U}$ : mechanical residual vector (equilibrium equation), $R_{T}$ : thermal residual vector (energy equation) and $\Delta$ indicates incremental values of the different variables.

The main features of this staggered scheme are:

- $\Delta U$ and $\Delta T$ are computed separately,

- a proper interchange of variables between both subsystems is performed in order to calculate the coupling terms and the thermomechanical variable boundary conditions: $I_{T U}=[T]$ and $I_{U T}=\left[\epsilon, \epsilon^{p}, g_{n}\right]\left(g_{n}\right.$ being the normal gap existing between two bodies $\left.{ }^{[9]}\right)$ are the thermalmechanical and mechanical-thermal interchanges, respectively. 
In particular, when $I_{T U}$ only exists, the problem is said to be thermally unidirectional coupled (TUC). For this case, it should be noted that $n_{\text {iter }}=1$ and $I_{T U}$ contains converged temperature values.

When both interchanges $I_{T U}$ and $I_{U T}$ exist, the problem is bidirectional coupled (BC). Within this class of solution strategy, different schemes can be proposed [9]. Specifically, the so called IterativeConverged-Consecutive strategy (It-Co-Ce) has been tested and used in different problems [9]. It basically consists in performing the interchanges $I_{T U}$ and $I_{U T}$ (both containing "locally" converged values) iteratively at the same time step in a consecutive form: the thermal problem (energy equation) is solved first followed by the mechanical one (equilibrium equation). This is the usual form used in the numerical simulation of solidification problems [4,5,9]. It should be noted that the "global" thermomechanical iteration index $j_{G}$ varies when both interchanges are performed.

As mentioned above, severe non-linearities exist in this coupled problem. Therefore, a proper convergence criterion for stopping the iteration process of each problem has to be used [9]. The coupled thermomechanical problem is converged (global convergence) when local convergence is achieved for both (mechanical and thermal) problems [9].

The quadratic convergence of Newion-Raphson's method is obviously losi. Nevertheless, it should be noted that the right hand side contains an "exact" (within the numerical frame) evaluation of the thermal and mechanical residuals. Finally, numerical results show good convergence rates and reasonable number of iterations [9].

\section{NUMERICAL EXAMPLE: SOLIDIFTCATION TEST.}
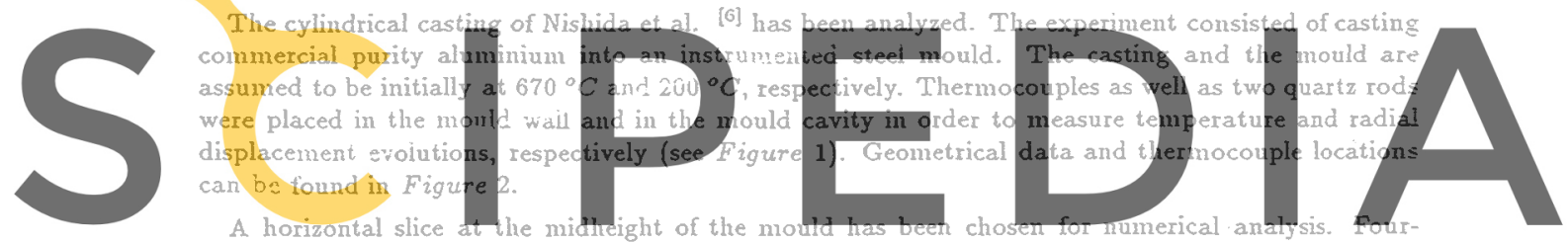

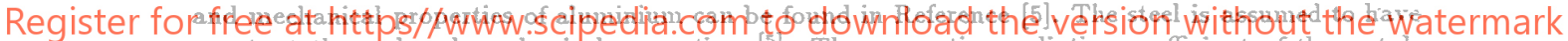
constant thermal and mechanical properties [5. The convection-radiation coefficient of the metalmould interface is gap-dependent, while the contact properties between aluminium and steel can be also found in Reference [9]. It should be noted that, for simplicity, a frictioniess condition has been considered.

As mentioned in Section 3, the system (3.1) has been solved using the BC-(It-Co-Ce) numerical strategy with a time step of $5 \mathrm{~s}$. In addition to the features already commented (see Section 3 ). this fact implies that the energy equation has been solved considering the coupling thermomechanical terms and taking into account the changes in the thermal boundary conditions due to the radial displacements of the specimen.

The temperature evolutions at different points of the mesh are plotted in Figure 3 and 4 . It is interesting to note the good agreement between the numerical results and the experimental ones.

In the solution of the mechanical problem, the bulk and shear moduli in the liquid has been computed using the elastic properties at the appropriate temperature. Considering that the shear modulus (computed using the tabulated material properties) in the surroundings of the melting point has a great value (it is evident that experimental data near the melting temperature is still lacking). the phase-change variable has been assumed to be:

$$
\rho_{T}(T)= \begin{cases}1 & ; \forall T<600.0^{\circ} \mathrm{C} \\ 1 \geq g_{T}(T) \geq 0 & ; 600.0^{\circ} C \leq \forall T \leq 660.0^{\circ} \mathrm{C} \\ 0 & ; \forall T>660.0^{\circ} \mathrm{C},\end{cases}
$$


where $g_{T}$ is a smooth cubic function of $T$ with zero slope for $T=600.0^{\circ} \mathrm{C}$ and $T=660.0^{\circ} \mathrm{C}$. Using this definition of $\rho_{T}$ and the mentioned material properties, the shear modulus increases in a smooth form (over a temperature range of $60^{\circ} \mathrm{C}$ ) in the material evolution from liquid to solid.

Figure 5 shows the radial displacement evolution of the mould-metal interface. Similarly, Figure 6 plots the same evolution for a point situated in the mould $1 \mathrm{~mm}$ from the inner surface. The progressive change in contact conditions, causing the convection-radiation coefficient to decrease significantly, can clearly be noted.

\section{CONCLUSIONS.}

Some important aspects of a thermomechanical constitutive model to simulate the solidification problem in casting has been presented. The model takes into account the different states of the solidifying material by introducing a new phase change variable, some internal plastic variables and a temperature-dependent constitutive law. Besides, full thermomechanical coupling terms and variable mechanical and thermal boundary conditions have been considered.

The model has been implemented into a finite element code. An enhanced staggered scheme has been proposed and used in order to solve the highly non-linear fully coupled finite element equations. Finally, the numerical example analyzed shows the robustness of the approach.

\section{ACKNOWLEDGEMENTS.}

The support provided by BRIE/EURAM Project no BE-4596 under contract n을 BRU-0443 is gratefully acknowledged.
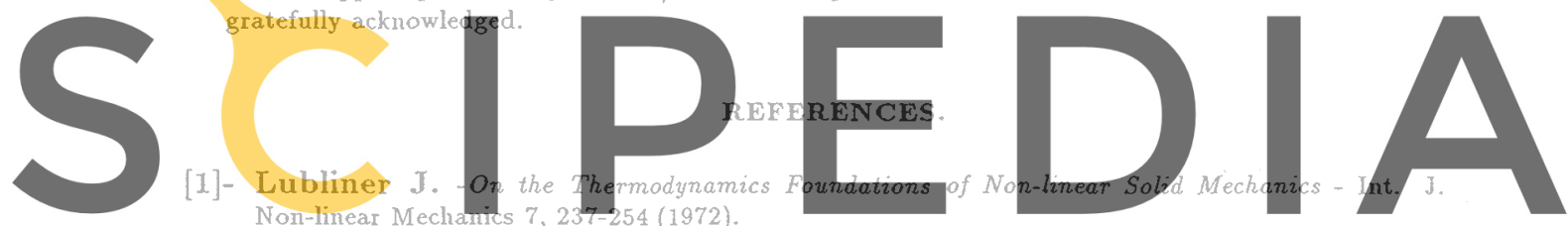

[2]- Brulhns O. and Služalec A. - Thermal Effects in Thermoplastic Metal with Internal Voriables

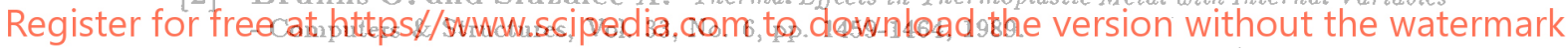

[3]- Armero F, and Simo J. - A New Unconditionally Stable Fractional Step Method for NonLinear Coupled Thermomechanical Problems - Tntenational Journal for Numerical Methods in Engineering, Vol. 35, 737-766 (1992).

[4]- Zabaras N., Ruan Y. and Richmond O. -Front Tracking Thermomechanical Model for Hypoelastic-Viscoplastic Behavior in a Solidifying Body-Computer Methods in Applied Mechanics and Engineering 81 (1990), 333-364, North-Holland.

[5]- Smelser R. and Richmond O. - Constitutive Model Effects on Stresses and Deformations in a Solidifying Circular Cylinder - Modeling of Casting and Welding Processes IV, 1988.

[6]- Nishida Y., Droste W. and Engler S. - The Air-Gap Formation Process at the Casting Mold Interface and the Heat Transfer Mechanism through the Gap-Metallurgical Transactions B, $17 \mathrm{~B}$ (1986) 833-844.

[7]- Celentano D., Oller S. and Oñate E. -A Constitutive Thermomechanical Model for Solidification of Metals - Journées Numériques de Besançon: Les problemes de changement de phase, pp. 19-30, Septembre 1991.

[8]- Celentano D., Oller S. and Oñate E. - A Plastic Constitutive Model to Simulate the Solidification in Casting Problems - Proceedings of Complas III, pp. 1089-1102. R. Owen, E. Oñate and E. Hinton (Eds.). Pineridge Press/CIMNE, 1992.

[9]- Celentano D. - A Thermomechanical Model for Solidification Problems in Metals - Ph.D. Thesis (in course), U.P.C., Barcelona, Spain, 1992. 

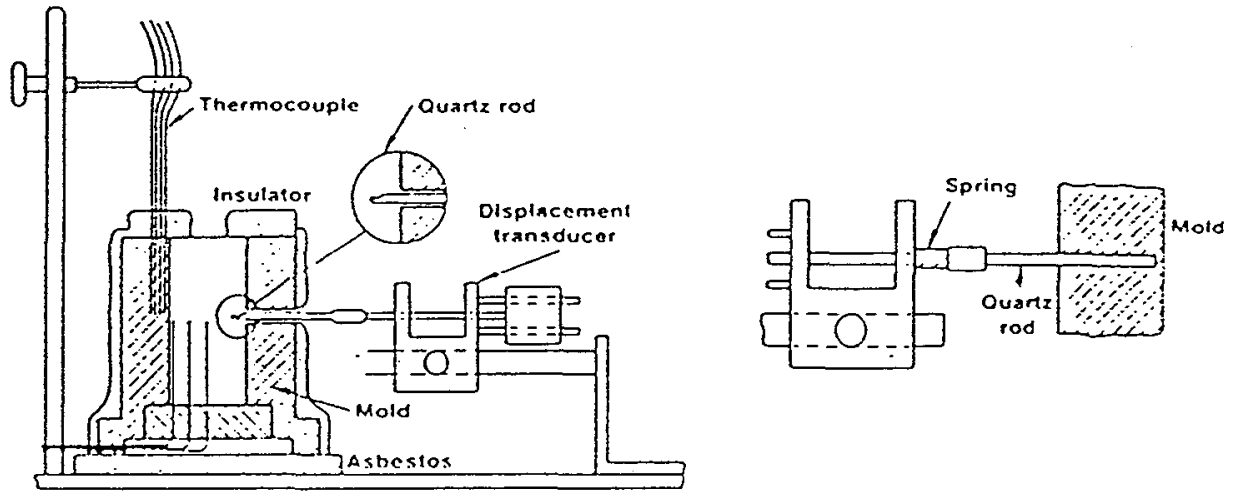

Figure 1. Solidification test: schematic of the experimental setup of Nishida et al. [6].
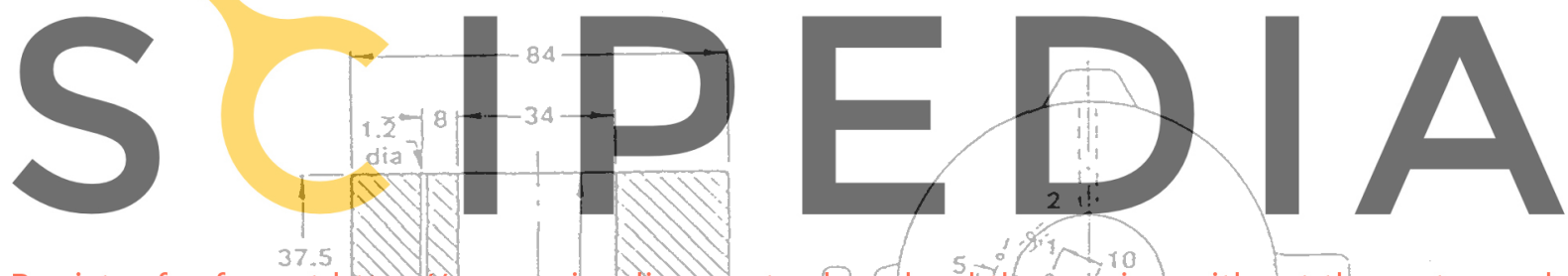

Register for free at htths s xwww.scipedia.com to dawnloaditheversion-without the watermark
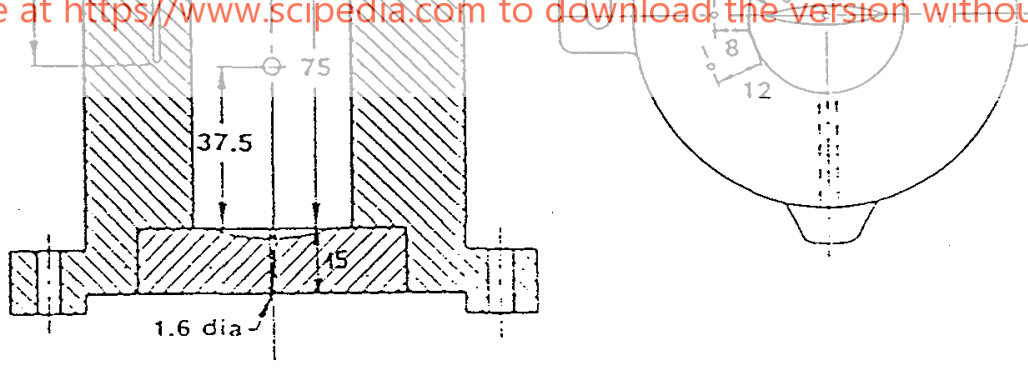

Figure 2. Solidification test: thermocouples locations in the mould and casting of the Nishida et al. [6] experiment. 

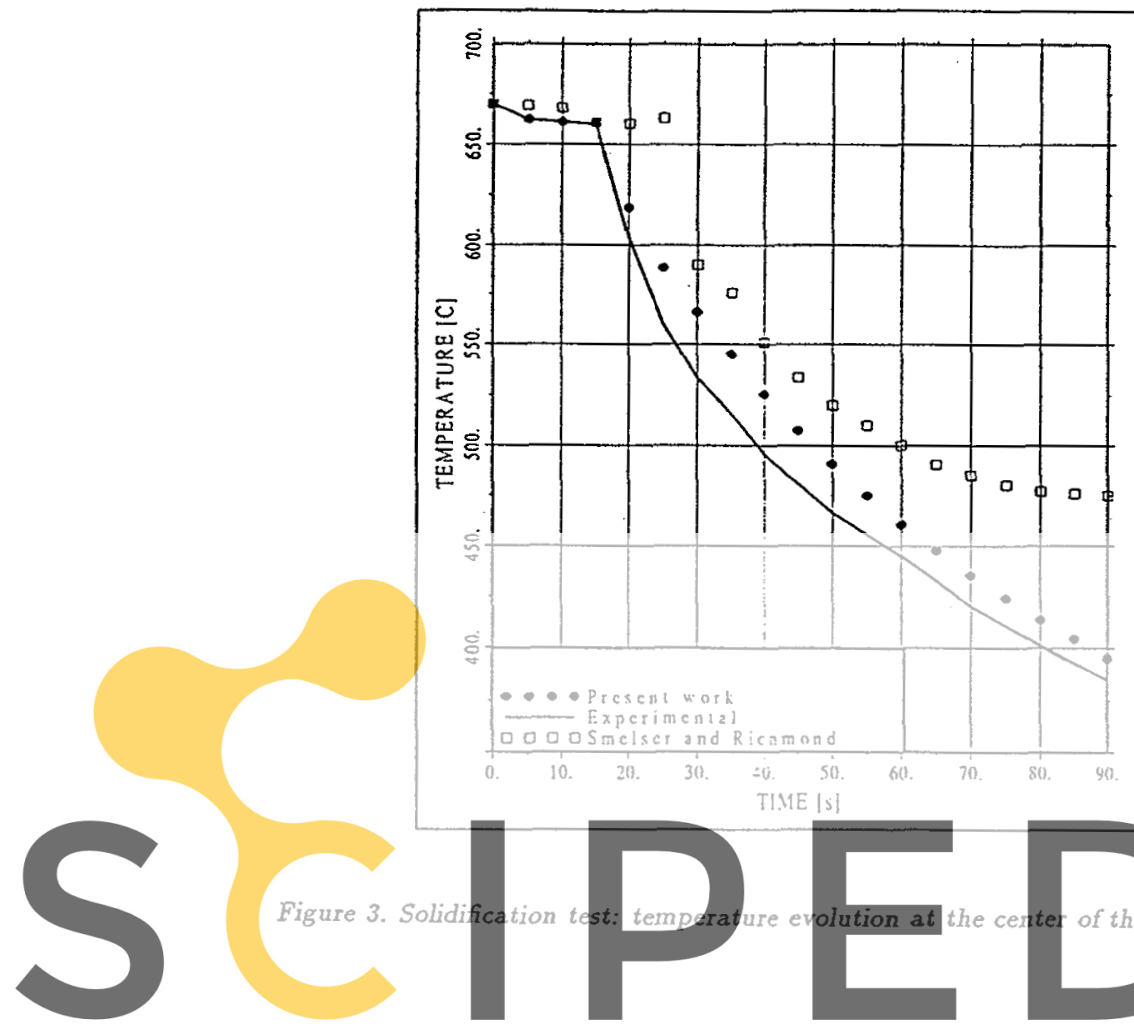

Register for free at https//vijww.\$cipedia.com to downtoad the version without the watermark

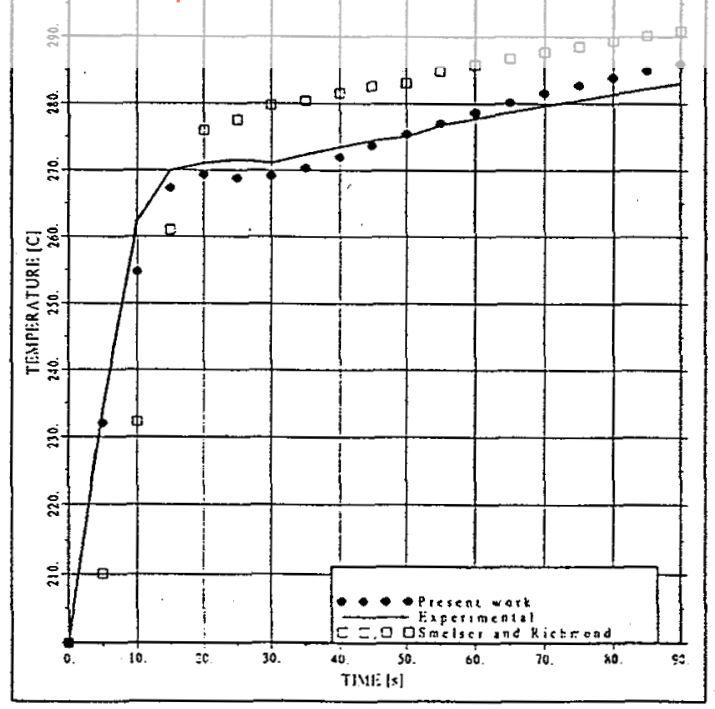

Figure 4. Solidification test: temperature evolution at a point situated $8 \mathrm{~mm}$ from the inner mould surface. 


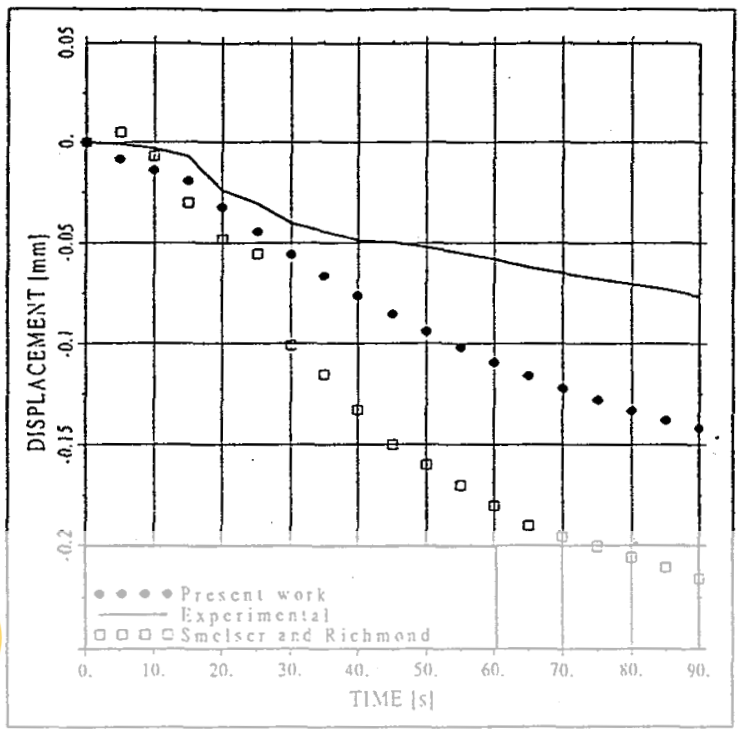

Figure 5. Solidification test: radial displacement evolution at the casting surface.
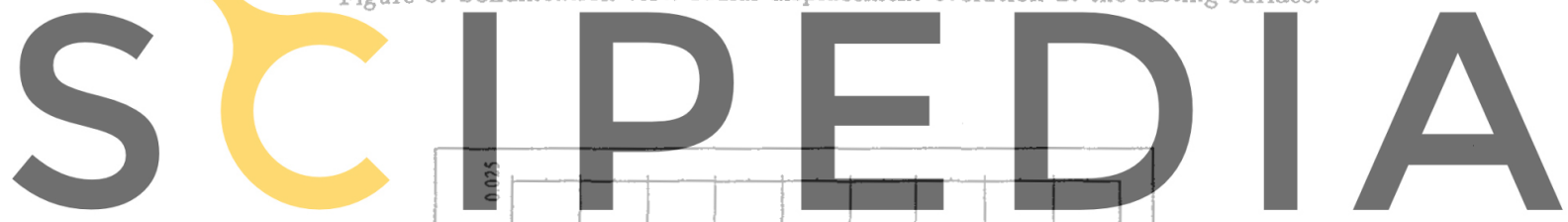

Register for free at https/ywww.scipedia.com to download the version without the watermark

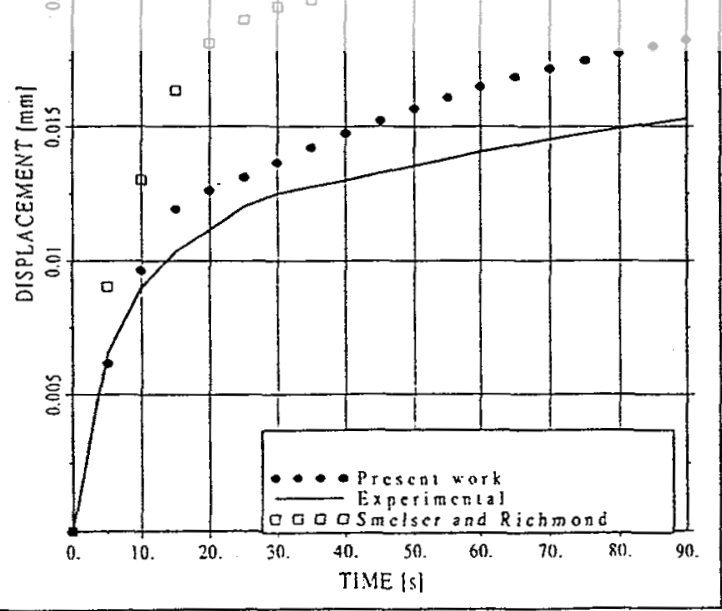

Figure 6. Solidification test: radial displacement evolution at a point situated $1 \mathrm{~mm}$ from the inner mould surface. 